\title{
An interesting case report of Morvan's syndrome from the Indian subcontinent
}

\author{
B. K. Bajaj, S. Shrestha \\ Neurology Division, Department of Internal Medicine, B. P. Koirala Institute of Health Sciences, Dhiı an, Nepal
}

\begin{abstract}
The French physician Augustine Marie Morvan first used the term 'la chorée fibrillare' to describe a syndrome characterized by peripheral nerve hyperexcitability, dysautonomia, insomnia and fluctuating delirium. There are no published reports of the condition from the Indian subcontinent. We report the first such case from the region. Our patient, a 24-year-old male, presented with easy fatigability and stiffness in both the calves for 18 months; continuous twitching of muscles of all four limbs and jaw for two months; hyperhydrosis, palpitations, urinary symptoms, burning dysesthesia in hands, insomnia and abnormal sleep behavior for about a month. Patient had bilateral hyper-reflexia with extensor plantar on the rigri. and equivocal response on the left. Electromyogifichy revealed continuous muscle fiber activity. Thyroid finction test, electroencephalography, computerized to.nugraphy scan (head) and routine cerebrospinal fluid an alysis were normal. The patient showed marke $\lambda$ clinics and electrophysiological improvement or predniso!ant along with symptomatic therapy over the $n \in X$ two months.
\end{abstract}

Key words: Dysautonomia, L Lauss' syndrome, limbic encephalitis, Morvan's syndr(im, $=$, syndrome of continuous muscle fiber activity

\section{Introduction}

The French physician Augustine Marie Morvan described a syndrome consisting of muscle twitching, dysautonomia, insomnia and fluctuating delirium by the name of 'la chorée fibrillare' in the late $19^{\text {th }}$ century. ${ }^{[1]}$ The clinical presentation is now better known as Morvan's syndrome. Over the last more than 100 years, various French and English authors have reported the condition. There are no published descriptions of the clinical entity from the Indian subcontinent. We report the first such case from the region.

\section{Case Report}

A 24-yeai cld male ro in Eastern Nepal while working as a spray painter in : $\mathrm{r}_{1}$ e Middle East, felt insidious onset excessive tiredness, stiffness and cramps in both the legs leading to pro, ressive inability to stand and walk. The intolerahle à scomfort and sense of stiffness led to a nearly bedridc'n state. About 16 months after the onset, he noticad continuous twitching of the muscles of the lower lirai s followed by the upper limbs and right jaw without associated weakness or wasting. One month later, he started feeling almost continuous palpitations and excessive sweating all over the body. He had to change his dress and bed-sheets three to four times daily as they would get drenched with sweat. He started having difficulty in falling asleep and his duration of sleep decreased to about $2-4 \mathrm{~h} / 24 \mathrm{~h}$. He behaved peculiarly during sleep. In the middle of sleep, the patient would start singing songs and walk out of bed. During these times, he stopped responding to family members appropriately. Next morning he would have no recollection of events. He earned himself the reputation of an 'FM radio' in his family due to his singing behavior. He started feeling burning dysesthesia and hyperesthesia in both the hands and feet without any complain of associated sensory loss. The patient developed increased diurnal and nocturnal urinary frequency and hesitancy without urinary retention, urgency or incontinence. On examination, there was resting tachycardia (Pulse rate $=130 / \mathrm{min}$ ) and supine blood pressure of 140/90 mmHg, with borderline postural drop. The other bedside tests of cardiac autonomic functions were within normal limits. Both the hands of the patient were swollen with scaly nonerythematous overlying skin without any change in temperature. Detailed chest, cardiovascular and abdominal examination

The manuscript was presented at the XIII Annual Conference of Indian Academy of Neurology, IANCON 2005 held at Jaipur from 23/09/05-25/09/05 
revealed no abnormality. Neurological assessment revealed a conscious visibly 'vexed' cooperative attentive individual with normal memory, language functions, praxis, gnosis and cranial nerve examination. Motor examination revealed continuous twitching in bilateral gastrocnemii, quadriceps femoris, biceps brachii and right masseter. The patient had normal muscle tone and power, bilaterally exaggerated deep tendon reflexes, and extensor plantar response on the right and equivocal plantar on the left. Hyperalgesia and hyperesthesia were noted below the knee and distal to the wrist joints bilaterally. The patient had an awkward gait and it appeared as if he was walking on hot coal. Nerve conduction study (motor and sensory) demonstrated normal latencies, amplitudes and conduction velocities in all the four limbs. Electromyography (EMG) revealed continuous fasciculations, multiplets and neuromyotonia in bilateral gastrocnemii, biceps brachii, quadriceps femoris and right masseter [Figures 1 and 2]. Awake electroencephalography (EEG) and computerized tomography scan (head) were

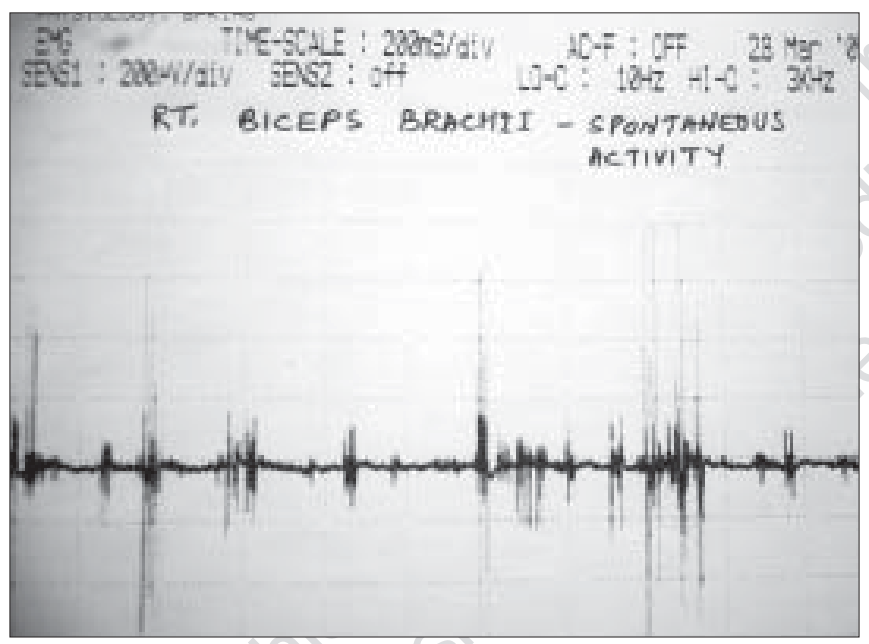

Figure 1: Spontaneous activity recorded from right biceps brachii prior to treatment showing multiplets

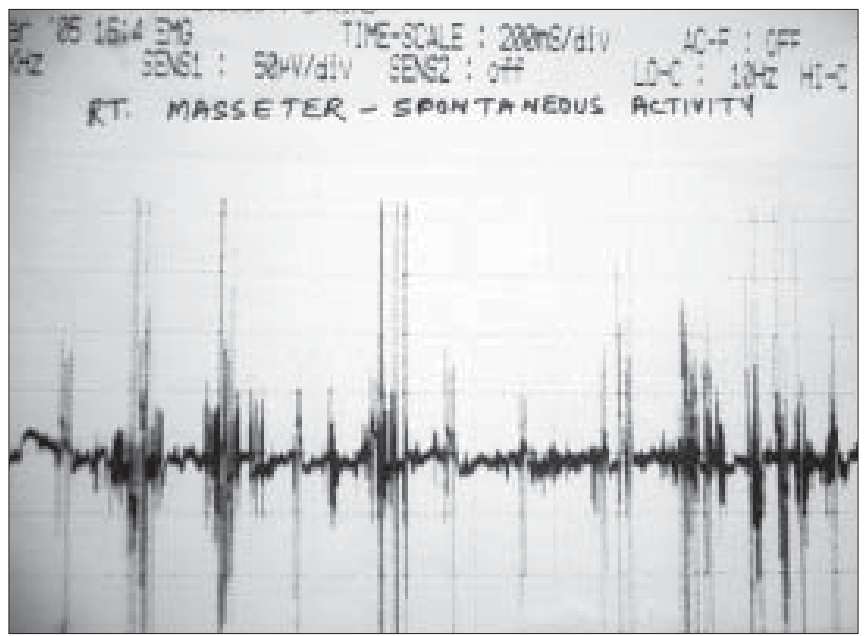

Figure 2: Continuous spontaneous activity including multiplets recorded from right masseter prior to treatment found to be normal. Lumbar puncture revealed normal cerebrospinal fluid protein, glucose and cells. Thyroid function test, ELISA for HIV and routine tests like hemogram, peripheral blood picture, erythrocyte sedimentation rate, liver function tests, kidney function tests, serum electrolytes, chest X-ray and ultrasoundabdomen were found to be normal. Electrocardiogram confirmed sinus tachycardia. Based on the clinical and laboratory findings, the patient was diagnosed to have Morvan's syndrome and was started on prednisolone 1 $\mathrm{mg} / \mathrm{kg}$ body wt. with carbamazepine, propanolol and clonazepam. Initially, the patient's symptoms seemed not to respond to any of these measures. Sleep was not helped by even $2 \mathrm{mg} / \mathrm{d}$ of clonazepam. He was started on amitriptyline and clonazepam was withdrawn but with little relief. The patient was observed and after about two weeks of drugs, the patient started showing signs of improvement with decreased stiffness and spontaneous muscle activity and improved sleep. After another seven to 10 days, his abnormal sleep behavior disappeared completely. He was continued on prednisolone, propanolol, carbamazepine and amitriptylin for another month with significant improvement. When the patient presented about six weeks later, he was almost free of discomfort, palpitations, insomnia, urinary symptoms, burning sensation and swelling of hands with absence of the "FM radio" behavior. However, spontaneous activity still continued albeit to a markedly reduced degree [Figure 3].

\section{Discussion}

There are about 14 reported cases of Morvan's syndrome (about 12 cases estimated by Joseph et $a I^{[1]}$ and two additional cases ${ }^{[2,3]}$ in the English literature. Our patient presented with widespread neurological involvement affecting the peripheral nervous system (muscle twitching, neuromyotonia), autonomic nervous system (resting tachycardia, borderline postural hypotension,

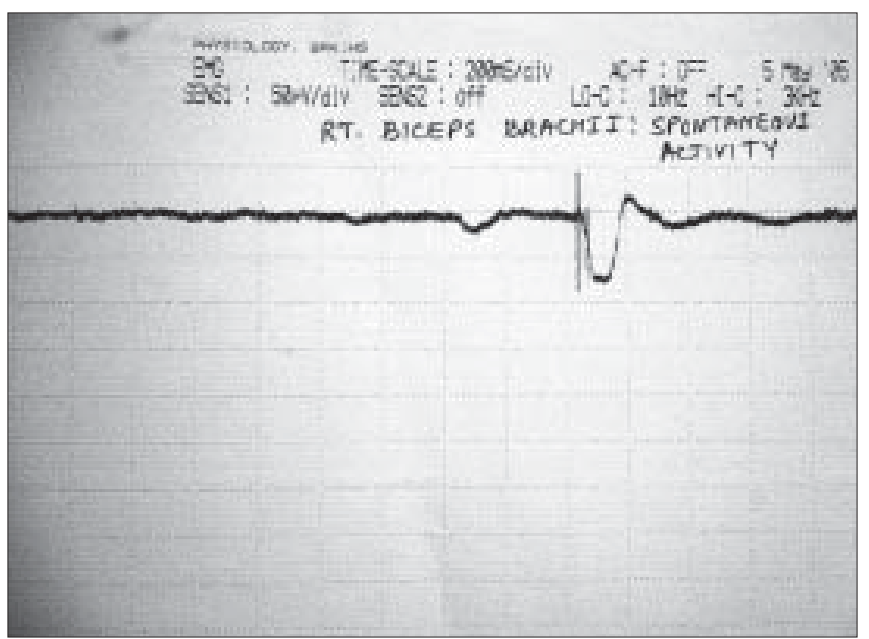

Figure 3: Markedly decreased spontaneous activity recorded from right biceps brachii about five weeks after start of treatment 
hyperhydrosis, urinary symptoms) and central nervous system (insomnia, abnormal sleep behavior, diffuse hyper-reflexia, extensor/equivocal plantar response).

Besides Morvan's syndrome, peripheral nerve hyperexcitability is a feature of other conditions like Isaacs' syndrome which presents as continuous muscle fiber activity with myokymia, muscle stiffness at rest, cramps and absent deep tendon reflexes. ${ }^{[4]}$ The association of spontaneous muscle fiber activity with additional features mentioned above sets Morvan's syndrome apart from Issac's syndrome. The differential diagnosis of Morvan's syndrome includes limbic encephalitis and fatal familial insomnia. Amnesia, seizures and mesial temporal lobe structural abnormalities are features of limbic encephalitis while myokymia, hyperhydrosis and insomnia favor Morvan's syndrome. ${ }^{[1]}$ Our patient had classical EMG findings and normal routine awake EEG. Polysomnography could not be undertaken due to lack of facility at our center. Polysomnographic observations in Morvan's syndrome include completely disrupted sleep structure with absent sleep cycles. ${ }^{[5]}$ Studies have shown subtly decreased metabolism on positron emission tomography (PET) and single photon emission computed tomography (SPECT) in the left inferior frontal and left temporal lobes. ${ }^{[1]}$

The pathophysiology of Morvan's syndrome is not established. Increased serum concentration of heavy metals has been reported by some authors. ${ }^{[6]}$ Our patient worked as a spray painter in the Middle East for about six months prior to onset. While screening for toxic metals might have been informative, we could not do it at our center. Recent descriptions of the syndrome as paraneoplastic manifestation of tumors like thymoma and small cell carcinoma and, often reported association with autoimmune thyroiditis and myasthenia gravis favor the autoimmune pathogenesis. ${ }^{[-9]}$ Detection of antibodies against voltage gated potassium channe ${ }^{[8,9]}$ and response of symptoms to immunomodulation ${ }^{[8,10]}$ further corroborates the autoimmune hypothesis. We could not determine the antibody levels at our institute because of lack of the facility.

Understandably, the therapy of the disorder is also not well established. There are mostly anecdotal reports about its treatment. We found the combination of carbamazepine, amitriptyline and prednisolone helpful in our patient. Some authors have reported improvement with use of intravenous immunoglobulins and plasmapheresis along with symptomatic therapy. Insomnia is recognized as a tough symptom to treat. While our patient's insomnia and sleep behavior did not respond to benzodiazepines, he started showing signs of improvement about two to three weeks after the use of the combination of prednisolone, amitriptyline and carbamazepine. Possibly, it was the steroid which resulted in the improvement of the patient's sleep. Loscher et al ${ }^{[3]}$ reported improvement with carbamazepine when immunomodulatory therapy with intravenous immunoglobulin therapy failed. Interestingly, spontaneous improvement can occur in some patients. The apparently vague sensory complains with strange fluctuating behavioral abnormality may lead to first visit to a psychiatrist. Careful history covering other aspects like presence of dysautonomia, sleep disturbance and EMG studies can help reach the correct diagnosis. Intensive and sometimes repeated evaluations for underlying tumor are warranted in these patients in view of reported paraneoplastic associations. Newer modalities like PET, SPECT and functional magnetic resonance imaging along with evaluation of antibody levels in the blood are likely to lead to better understanding of the syndrome and its relation with other disorders with continuous muscle fiber activity.

\section{References}

1. Josephs KA, Silber MH, Fealey RD, Nippoldt TB, Auger RG, Vernino S. Neurophysiologic studies in Morvan syndrome. J Clin Neurophysiol 2004;21:440-5.

2. Cottrell DA, Blackmore KJ, Fawcett PR, Birchall D, Vincent A, Barnard S, et al. Sub-acute presentation of Morvan's syndrome after thymectomy. J Neurol Neurosurg Psychiatry 2004;75:1504-5.

3. Loscher WN, Wanschitz J, Reiners K, Quasthoff S. Morvan's syndrome: Clinical, laboratory and in vitro electrophysiological studies. Muscle Nerve 2004;30:157-63.

4. Issacs H. A syndrome of continuous muscle fiber activity. J Neurol Neurosurg Psychiatry 1964;24:319-25.

5. Vetrugno R, Liguori R, Provini F, Plazzi G, Montagna P. Continuous motor unit activity syndromes: A video-polysomnographic study. Clin Neurophysiol 2005;116:2533-41.

6. Haug BA, Schoenle PW, Karch BJ, Bardosi A, Holzgraefe M Morvan's fibrillary chorea. A case with possible manganese poisoning. Clin Neurol Neurosurg 1989;91:53-9.

7. Halbach M, Homberg V, Freund HJ. Neuromuscular, autonomic and central cholinergic hyperactivity associated with thymoma and acetylcholine receptor-binding antibody. J Neurol 1987;234:433-6.

8. Lee EK, Maselli RA, Ellis WG, Agius MA. Morvan's fibrillary chorea: A paraneoplastic manifestation of thymoma. J Neurol Neurosurg Psychiatry 1998;65:857-62.

9. Barber PA anderson NE, Vincent A. Morvan's syndrome associated with voltage gated $\mathrm{K}^{+}$channel antibodies. Neurology 2000;54:771-2.

10. Liguori R, Vincent A, Clover L, Avoni P, Plazzi G, Cortelli P et al. Morvan's syndrome: Peripheral and central nervous system and cardiac involvement with antibodies to voltage-gated potassium channels. Brain 2001;124:2417-26.

Accepted on 10-07-2006

Source of Support: Nil, Conflict of Interest: None declared. 